\title{
Violation of self-similarity in the expansion of a one-dimensional Bose gas
}

\author{
P. Pedri, ${ }^{1,2}$ L. Santos, ${ }^{1}$ P. Öhberg, ${ }^{3}$ and S. Stringari ${ }^{2}$ \\ ${ }^{1}$ Institut für Theoretische Physik, Universität Hannover, D-30167 Hannover, Germany \\ ${ }^{2}$ Dipartimento di Fisica, Università di Trento and BEC-INFM, I-38050 Povo, Italy \\ ${ }^{3}$ Department of Physics, University of Strathclyde, Glasgow G4 ONG, Scotland
}

(Received 29 April 2003; published 1 October 2003)

\begin{abstract}
The expansion of a one-dimensional Bose gas after releasing its initial harmonic confinement is investigated employing the Lieb-Liniger equation of state within the local-density approximation. We show that during the expansion the density profile of the gas does not follow a self-similar solution, as one would expect from a simple scaling ansatz. We carry out a variational calculation, which recovers the numerical results for the expansion, the equilibrium properties of the density profile, and the frequency of the lowest compressional mode. The variational approach allows for the analysis of the expansion in all interaction regimes between the mean-field and the Tonks-Girardeau limits, and in particular shows the range of parameters for which the expansion violates self-similarity.
\end{abstract}

DOI: 10.1103/PhysRevA.68.043601

PACS number(s): 03.75.Kk, 05.30.Jp

\section{INTRODUCTION}

The experimental achievement of Bose-Einstein condensation (BEC) [1] has aroused a large interest in the physics of ultracold atomic gases. Among the topics related to this field, the physics of low-dimensional atomic gases has recently attracted significant attention. The development of the trapping techniques has allowed for the realization of very anisotropic geometries, where the confinement is so strong in one or two dimensions that at low temperatures the transversal motion is "frozen," and does not contribute to the dynamics of the system. In this way two- [2-5] and onedimensional $[2,6,7]$ systems have been accomplished. Lowdimensional gases present significantly different properties compared to the three-dimensional ones. A remarkable example is provided by the existence of quasicondensation [8-11], whose effects have been recently observed experimentally [12].

During the last years, the one-dimensional (1D) Bose gases have been the subject of growing interest, in particular, the limit of impenetrable bosons [13], which behave to a large extent as a noninteracting Fermi system, acquiring some remarkable properties. The conditions for the experimental realization of strongly correlated $1 \mathrm{D}$ gases are rather restrictive $[8,14]$, since a large radial compression, a sufficiently small density, and eventually a large scattering length are needed. Fortunately, recent experimental developments have opened perspectives in this sense. Especially interesting is the possibility to modify at will the interatomic interactions by means of Feshbach resonances [15] and the capability of loading an atomic gas in an optical lattice [16].

From the theoretical side, the physics of 1D Bose gases was first investigated by Girardeau [13], who considered the limit of impenetrable bosons, also called Tonks-Girardeau (TG) gas, pointing out a nontrivial relation with the physics of ideal Fermi gases. This analysis was later extended by Lieb and Liniger [21], who solved analytically the problem for any regime of interactions, using Bethe ansatz. Yang and Yang [22] extended the analysis including finite temperature effects. Recently, the experimental accessibility of trapped gases have encouraged the investigation of the harmonically trapped case. The Bose-Fermi (BF) mapping has been employed to the case of an inhomogeneous gas in the TG limit [23]. However, there is unfortunately, to the best of our knowledge, no exact solution for arbitrary interaction strength in the case of trapped gases. The problem of the equilibrium of a trapped gas can be analyzed using a localdensity approximation and employing the Lieb-Liniger (LL) equation of state locally to evaluate the equilibrium density profiles [17]. A similar formalism has been recently employed to analyze the collective oscillations in the presence of harmonic trapping [18]. Both Refs. [17] and [18] have shown the occurrence of a continuous transition from the mean-field (MF) regime to the TG one as the intensity of the interaction is varied. Recently, Gangardt and Shlyapnikov [19] have discussed the stability and phase coherence of 1D trapped Bose gases. These authors have analyzed the local correlation properties and found that inelastic decay processes, such as three-body recombination, are suppressed in the TG regime, and intermediate regimes between MF and TG. This fact opens promising perspectives towards the accomplishment of strongly interacting 1D Bose gases with large number of particles. This analysis have been very recently extended to the case of finite temperatures [24].

The expansion of a one-dimensional Bose gas in a guide was analyzed in Ref. [20] by means of a hydrodynamic approach based on the local LL model. The expansion dynamics was shown to be different for different interaction strengths, and its analysis could be employed to discern between the TG and MF regimes. In particular, the self-similar solution is violated.

In this paper, we extend the analysis of Ref. [20] by introducing a variational approach, which permits us to study the asymptotic regime at large expansion times. This method is shown to be in excellent agreement with previous direct numerical simulations, and additionally permits us to recover the results of Refs. [17,18]. More importantly, our variational approach allows us to determine the regime of parameters for which the self-similarity of the expansion is violated.

The paper is organized as follows. In Sec. II we introduce 
the local LL model which we employ to analyze the expansion dynamics. In Sec. III we briefly discuss the numerical results obtained in Ref. [20]. In Sec. IV we present a variational approach which allows us to investigate in detail the expansion dynamics for arbitrary regimes of parameters. Finally we conclude in Sec. V.

\section{LOCAL LIEB-LINIGER MODEL}

We analyze in the following a dilute gas of $N$ bosons confined in a very elongated harmonic trap with radial and axial frequencies $\omega_{\rho}$ and $\omega_{z}\left(\omega_{\rho} \gg \omega_{z}\right)$. We assume that the transversal confinement is strong enough so that the interaction energy per particle is smaller than the zero-point energy $\hbar \omega_{\rho}$ of the transversal trap. In this way, the transversal dynamics is effectively frozen and the system can be considered as dynamically 1D. In this section we briefly review the formalism introduced in Ref. [17].

We assume that the interparticle interaction can be approximated by a $\delta$ function pseudopotential. Therefore the Hamiltonian that describes the physics of the 1D gas becomes

$$
\hat{H}_{1 \mathrm{D}}=\hat{H}_{1 \mathrm{D}}^{0}+\sum_{j=i}^{N} \frac{m \omega_{z}^{2} z_{i}^{2}}{2},
$$

where

$$
\hat{H}_{1 \mathrm{D}}^{0}=-\frac{\hbar^{2}}{2 m} \sum_{j=1}^{N} \frac{\partial^{2}}{\partial z_{j}^{2}}+g_{1 \mathrm{D}} \sum_{i=1}^{N-1} \sum_{j=i+1}^{N} \delta\left(z_{i}-z_{j}\right)
$$

is the homogeneous Hamiltonian in the absence of the harmonic trap, $m$ is the atomic mass, and $g_{1 \mathrm{D}}=-2 \hbar^{2} / m a_{1 \mathrm{D}}$. The scattering problem under one-dimensional constraints was analyzed in detail by Olshanii [14], and it is characterized by the one-dimensional scattering length $a_{1 \mathrm{D}}$ $=\left(-a_{\rho}^{2} / 2 a\right)\left[1-\mathcal{C}\left(a / a_{\rho}\right)\right]$, with $a$ the three-dimensional scattering length, $a_{\rho}=\sqrt{2 \hbar / m \omega_{\rho}}$ is the oscillator length in the radial direction, and $\mathcal{C}=1.4603 \ldots$. As shown by Lieb and Liniger [21], the homogeneous Hamiltonian $\hat{H}_{1 \mathrm{D}}^{0}$ can be diagonalized exactly by means of Bethe ansatz [25]. In the thermodynamic limit, a 1D gas at zero temperature with a given linear density $n$ is characterized by the energy per particle

$$
\epsilon(n)=\frac{\hbar^{2}}{2 m} n^{2} e(\gamma(n)),
$$

where $\gamma=2 / n\left|a_{1 \mathrm{D}}\right|$. The function $e(\gamma)$ fulfills

$$
e(\gamma)=\frac{\gamma^{3}}{\lambda^{3}(\gamma)} \int_{-1}^{1} g(x \mid \gamma) x^{2} d x,
$$

where $g(x \mid \gamma)$ and $\lambda(\gamma)$ are the solutions of the LL system of equations [21]

$$
g(x \mid \gamma)=\frac{1}{2 \pi}+\frac{1}{2 \pi} \int_{-1}^{1} \frac{2 \lambda(\gamma)}{\lambda^{2}(\gamma)+(y-x)^{2}} g(y \mid \gamma) d y
$$

$$
\lambda(\gamma)=\gamma \int_{-1}^{1} g(x \mid \gamma) d x
$$

We assume next that at each point $z$ the gas is in local equilibrium, with local energy per particle provided by Eq. (3). Then, one can obtain the corresponding hydrodynamic equations for the density and the atomic velocity

$$
\begin{gathered}
\frac{\partial}{\partial t} n+\frac{\partial}{\partial z}(n v)=0, \\
\frac{\partial}{\partial t} v+v \frac{\partial}{\partial z} v=-\frac{1}{m} \frac{\partial}{\partial z}\left[\mu_{l e}(n)+\frac{1}{2} m \omega_{z}^{2} z^{2}\right],
\end{gathered}
$$

where

$$
\mu_{l e}(n)=\left(1+n \frac{\partial}{\partial n}\right) \epsilon(n)
$$

is the Gibbs free energy per particle. The hydrodynamic description holds if the local chemical potential, related to the interaction energy in the homogeneous system, is much larger than the kinetic energy associated to the density modulations. It assumes, in particular, that the density of the gas varies smoothly within the typical distance fixed by the healing length (local-density approximation). Note that for the case of $n\left|a_{1 \mathrm{D}}\right| \rightarrow \infty$, one obtains $\mu_{l e}(n)=g_{1 \mathrm{D}} n$, retrieving the 1D Gross-Pitaevskii equation [26], whereas for the case $n\left|a_{1 \mathrm{D}}\right| \rightarrow 0$, one gets $\mu_{l e}(n)=\pi^{2} \hbar^{2} n^{2} / 2 m$, and the equation of Ref. [27] is recovered. The system has only one control parameter $[8,17,18]$, namely, $A=N\left|a_{1 \mathrm{D}}\right|^{2} / a_{z}^{2}$, where $a_{z}$ $=\sqrt{\hbar / m \omega_{z}}$ is the harmonic-oscillator length in the $z$ direction. The regime $A \gg 1$ corresponds to the MF limit, in which the stationary-state density profile has a parabolic form. On the other hand, the regime $A \ll 1$ corresponds to the TG regime, which is characterized by a stationary-state density profile with the form of a square root of a parabola.

\section{NUMERICAL RESULTS}

In Ref. [20], Eqs. (3), (5), (6), and (9) were employed to simulate numerically the expansion of a 1D gas in the framework of the hydrodynamic formalism. The expansion follows the sudden removal of the axial confinement, while the radial one is kept fixed. In particular, it was observed that during the expansion the density profile is well described by the expression

$$
n(z, t)=n_{m}(t)\left[1-\left(\frac{z}{R(t)}\right)^{2}\right]^{s(t)},
$$

where $n_{m}(t)$ provides the appropriate normalization, $R(t)$ is the radius of the cloud, and the exponent $s(t)$ takes the value $s(0)=1$ for an initial MF gas. The function $s(t)$ decreases monotonically in time, approaching an asymptotic value (see Fig. 1). Therefore, contrary to the expansion dynamics for a BEC [28-30], the self-similarity of the density profile is violated. 


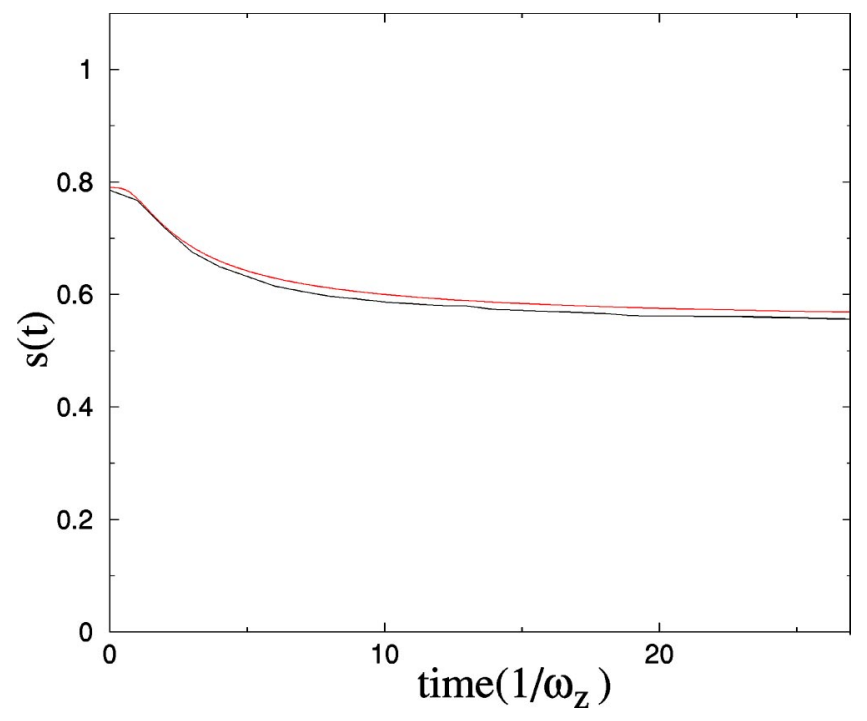

FIG. 1. Time evolution of the exponent $s(t)$ for $A=0.43, \omega_{\rho}$ $=2 \pi(20) \mathrm{kHz}$, and $N=200$ atoms $\left[\omega_{z}=2 \pi \times(1.8) \mathrm{Hz}\right.$ at $\left.t=0\right]$. Our variational result (dashed line) shows a very good agreement with the results obtained from the direct resolution of Eqs. (7) and (8).

At this point we discuss the physics behind this violation of the self-similarity. If the local chemical potential presents a fixed power-law dependence on the density, $\mu_{l e} \propto n^{\lambda}$, it is easy to show from the hydrodynamic equations (7) and (8) that there exists a self-similar solution of the form $n$ $=\left(n_{0} / b\right)\left[1-(z / b R)^{2}\right]^{1 / \lambda}$, where $\ddot{b}=\omega_{z}^{2} / b^{\lambda+1}$. For the particular case of the TG gas, the scaling law can be also obtained from the exact BF mapping [20]. However, since $\mu_{l e}$ is obtained from the LL equations, the dependence of $\mu_{l e}$ on $n$ is quadratic for a low density and linear for a large one. Therefore, $\mu_{l e}$ does not fulfill a fixed power-law dependence during the expansion, and the self-similarity is violated. In particular, as the expansion proceeds the whole system approaches the low-density regime, and consequently, the exponent $s$ decreases monotonically. In the following section, we analyze in more detail this effect.

\section{VARIATIONAL CALCULATION}

In this section, we complete our understanding of the expansion of a one-dimensional Bose gas in a guide by means of a variational ansatz using a Lagrangian formalism. The Lagrangian density for the system is of the form

$$
\mathcal{L}=-m n \frac{\partial \phi}{\partial t}-\frac{1}{2} m n\left(\frac{\partial \phi}{\partial z}\right)^{2}-\frac{1}{2} m \omega_{z}^{2} z^{2} n-\varepsilon(n) n,
$$

where the velocity field is defined as $v=\partial \phi / \partial z$. The equations of motion are obtained from the functional derivation of the action $\mathcal{A}=\int \mathcal{L} d t d z: \delta \mathcal{A} / \delta \phi=0$ (continuity equation), $\delta \mathcal{A} / \delta n=0$ (which after partial derivation with respect to $z$ provides the Euler equation). From the numerical results we have observed that the density is at any time well described by Eq. (10). Therefore, we assume the following ansatz for the density:

$$
n=\frac{C(s)}{b}\left(1-\frac{z^{2}}{R^{2} b^{2}}\right)^{s}
$$

where $b$ and $s$ are time-dependent variables, $R$ is the initial Thomas-Fermi radius, and $C(s)$ is related to the normalization to the total number of particles. For the $\phi$ field we consider the following form:

$$
\phi=\frac{1}{2} \alpha z^{2}+\frac{1}{4} \beta z^{4}
$$

where $\alpha$ and $\beta$ are time-dependent parameters. We stress at this point that in the analysis of the self-similar expansion of a BEC [28-30] a quadratic ansatz (in z) for the $\phi$ field provides the exact solution. However, for the problem under consideration in this paper, it is necessary to include higherorder terms to account for the violation of the self-similarity. We have checked that terms of higher order than $z^{4}$ introduce only small corrections, and therefore we reduce to the form of Eq. (13).

We are interested in the dynamics of the parameters $b$ and $s$, related to the size and the shape of the cloud, respectively. Integrating the Lagrangian density in $z, L=\int \mathcal{L} d z$, one finds a Lagrangian for the above-mentioned parameters:

$$
\begin{aligned}
L(\dot{\alpha}, \alpha, \dot{\beta}, \beta, b, s)= & \frac{m N R^{2}}{2}\left\{-\frac{\dot{\alpha} b^{2}}{2 s+3}-\frac{3}{2} \frac{\dot{\beta} b^{4}}{(2 s+5)(2 s+3)}\right. \\
& -\frac{\alpha^{2} b^{2}}{2 s+3}-2 \frac{\alpha \beta b^{4}}{(2 s+5)(2 s+3)} \\
& \left.-\frac{\beta^{2} b^{6}}{(2 s+7)(2 s+5)(2 s+3)}-\frac{b^{2} \omega_{z}^{2}}{2 s+3}\right\} \\
& -\int d z n \epsilon(n) .
\end{aligned}
$$

We perform a gauge transformation [31]

$$
L(t, q, \dot{q}) \rightarrow L(t, q, \dot{q})+\frac{d}{d t} g(t, q),
$$

where

$$
g(t, q)=\frac{m N R^{2}}{2}\left\{-\frac{\alpha b^{2}}{2 s+3}-\frac{3}{2} \frac{\beta b^{4}}{(2 s+5)(2 s+3)}\right\} .
$$

The resulting Lagrangian is of the form $L$ $=L(\alpha, \beta, \dot{b}, b, \dot{s}, s)$. Imposing the conservation laws $\partial L / \partial \alpha$ $=\partial L / \partial \beta=0$, we obtain a Lagrangian depending only on the two relevant parameters $s$ and $b$, of the form $L=K-V$, where

$$
\begin{aligned}
\frac{V}{E_{1 \mathrm{D}}} & =\left(\frac{B(b, s)}{f_{0}(s)}+\frac{A^{2} b^{2}}{\left[\eta_{0} f_{0}\left(s_{0}\right)\right]^{2}} \frac{1}{2 s+3}\right), \\
\frac{K}{E_{1 \mathrm{D}}} & =\frac{A^{2}\left(M_{11} \dot{b}^{2}+2 M_{12} \dot{b} \dot{s}+M_{22} \dot{s}^{2}\right)}{\left[\eta_{0} f_{0}\left(s_{0}\right)\right]^{2}(2 s+3)},
\end{aligned}
$$




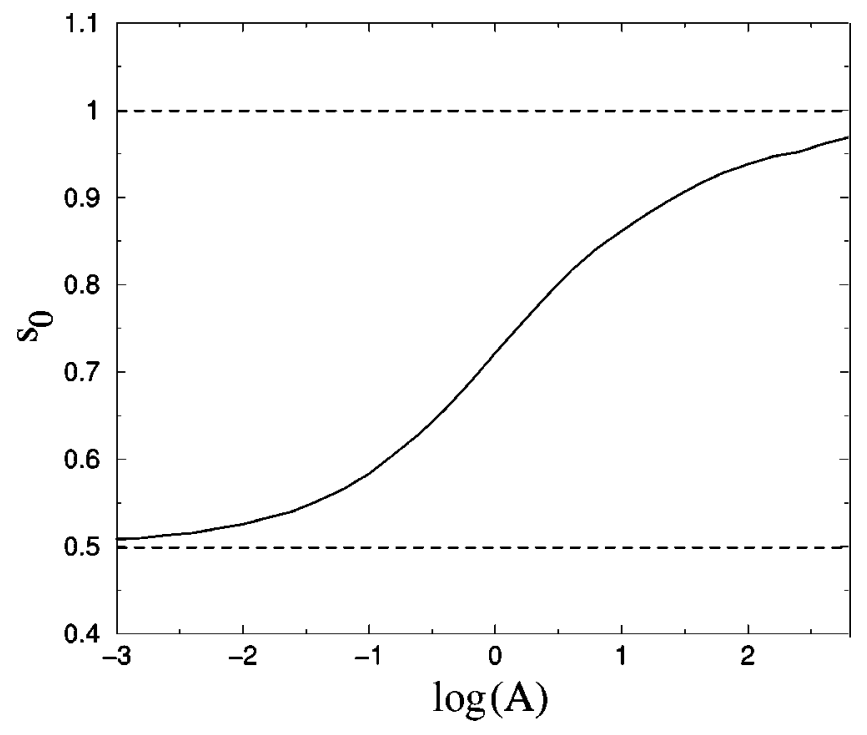

FIG. 2. Equilibrium values (at $t=0$ before opening the trap) of the exponent $s$, as a function of the parameter $A$. The dashed lines denote the MF limit, $s_{0}=1$, and the TG one, $s_{0}=1 / 2$.

where $E_{1 \mathrm{D}}=\hbar^{2} / 2 m\left|a_{1 \mathrm{D}}\right|^{2}$ is the typical energy associated with the interatomic interactions. In Eq. (17), we use the function $B(b, s)=\int d y\left(1-y^{2}\right)^{s} \epsilon(n(y)) / E_{1 \mathrm{D}}$, where we integrate over the rescaled axial coordinate $y=z / R b$. In Eqs. (17) and (18), we define the dimensionless central density $\eta_{0}=n_{0}\left|a_{1 \mathrm{D}}\right|$, where $n_{0}$ is the initial central density and the parameter $s_{0}=s(t=0)$. We have additionally employed the auxiliary functions $f_{n}(s)=\int y^{n}\left(1-y^{2}\right)^{s} d y$, and the coefficients

$$
\begin{gathered}
M_{11}=1, \\
M_{12}=\frac{-b}{2 s+3}, \\
M_{22}=\frac{b^{2}\left(121+186 s+96 s^{2}+16 s^{3}\right)}{4(s+1)(2 s+3)^{2}(2 s+5)^{2}} .
\end{gathered}
$$

From the Lagrangian $L$, one obtains the corresponding Euler-Lagrange equations for the parameters $b$ and $s$. In order to find the initial conditions $\eta_{0}$ and $s_{0}$ for the expansion, we have numerically minimized the potential $V$ in the presence of the harmonic trap for different values of $A$, assuming $b=1$ (see Fig. 2). When $A \gg 1, s_{0}$ tends to 1 , as expected for the MF case. On the contrary, when $A \ll 1, s_{0}$ tends to $1 / 2$ (TG profile). As expected from Ref. [17], for $A \ll 1, \eta_{0}$ $\propto A^{1 / 2}$, whereas for $A \gg 1$, the MF dependence $\eta_{0} \propto A^{2 / 3}$ is recovered.

Our variational approach allows us to calculate the lowest compressional mode, offering an alternative method as the one discussed in Ref. [18]. Expanding the potential $V$ around the equilibrium solution up to second order in $b$ (see Fig. 3), and neglecting for small oscillations the time dependence of $s$, we obtain

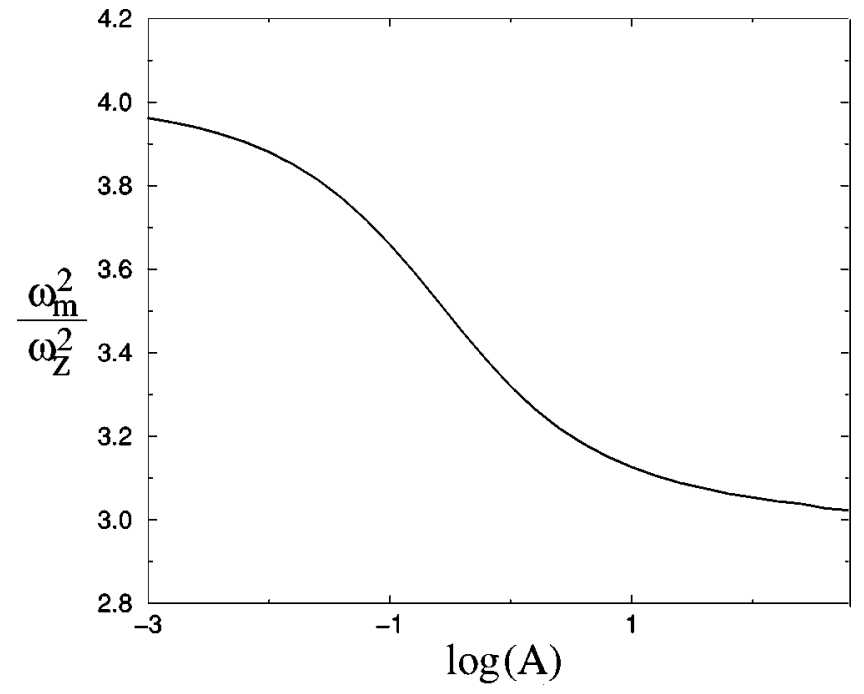

FIG. 3. Frequency of the lowest compressional mode as a function of the parameter $A$.

$$
\frac{\omega_{m}^{2}}{\omega_{z}^{2}}=1+\left.\frac{1}{2 A^{2}} \frac{\left[\eta_{0}^{2} f_{0}\left(s_{0}\right)\right]^{2}}{f_{2}\left(s_{0}\right)} \frac{\partial^{2} B}{\partial b^{2}}\right|_{b=1} .
$$

Our results show a continuous transition from the MF value, $\omega_{m}=\sqrt{3} \omega_{z}$, to the TG one, $\omega_{m}=2 \omega_{z}$, in excellent agreement with the results obtained by means of a sum rule formalism [18].

From the corresponding Euler-Lagrange equations, we have obtained the dynamics of $b(t)$ and $s(t)$. We have checked in all our calculations that the energy and number of particles remain a constant of motion. We have compared the variational results with our simulations based on the exact resolution of the hydrodynamic equations [20], obtaining an excellent agreement (see Fig. 1).

We have analyzed the asymptotic value $\dot{b}_{\infty}$ for different values of $A$. It is easy to obtain, that for a power-law dependence of the local chemical potential $\mu_{l e} \propto n^{\lambda}$, the derivative of the scaling parameter asymptotically approaches a value $\dot{b}_{\infty}=\sqrt{2 / \lambda} \omega_{z}$. Therefore a continuous transition from $\dot{b}_{\infty}$ $=\omega_{z}(\mathrm{TG})$ to $\dot{b}_{\infty}=\sqrt{2} \omega_{z}(\mathrm{MF})$ is expected. We recover this dependence from our variational calculations (see Fig. 4).

We have analyzed the behavior of $s$ during the expansion dynamics for different values of $A$. In particular, we have defined the asymptotic ratio $\xi=s_{\infty} / s_{0}$, with $s_{\infty}=s(t \rightarrow \infty)$ (see Fig. 5). Deeply in the TG regime $(A \ll 1)$ or in the MF one $(A \gg 1), \xi \simeq 1$, i.e., in those extreme regimes, the expansion is well described by a self-similar solution. However, for intermediate values, $\xi<1$, i.e., the expansion is not selfsimilar. The self-similarity is maximally violated in the vicinity of $A=1$, although $\xi$ departs significantly from 1 for a range $0.01 \leqq A \leqq 100$. The behavior at large $A$ can be understood as follows. If the gas is at $t=0$ deeply in the MF regime, the change of the functional dependence of $\mu_{l e}$ with the density occurs at very long expansion times, when the initial interaction energy of the gas has been fully transferred into kinetic energy. Therefore, for large values of $A$ the selfsimilarity is recovered. 


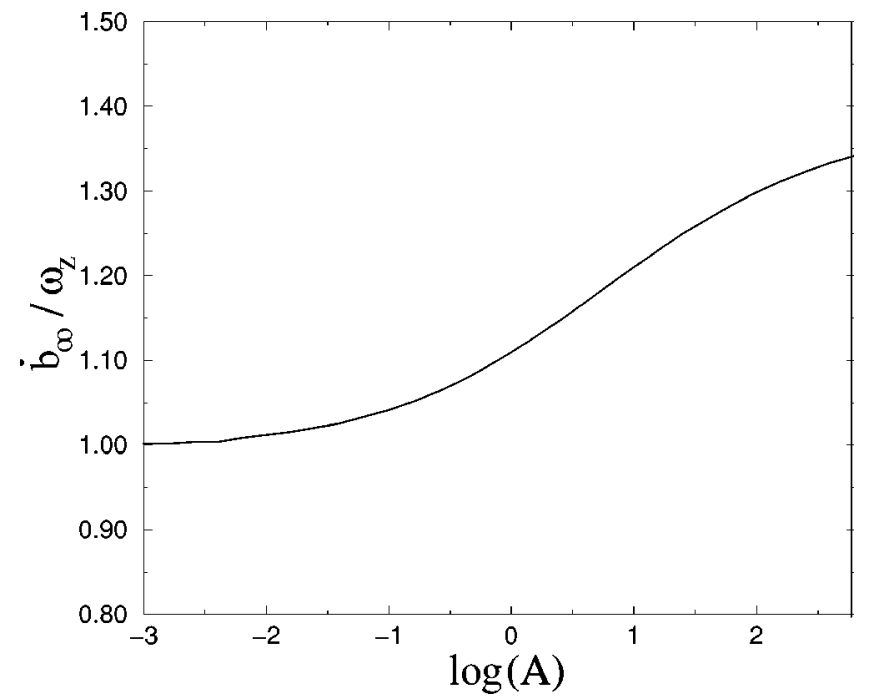

FIG. 4. Expansion velocity. Asymptotic value of $\dot{b}$ as a function of the parameter $A$.

\section{CONCLUSIONS}

In this paper, we have extended the analysis of Ref. [20] on the expansion dynamics of a one-dimensional Bose gas in a guide. We have shown that the expansion violates under certain conditions the self-similarity, and in this sense differs significantly from the expansion dynamics of a BEC. We have shown that the problem can be solved by employing the hydrodynamic approach and the local Lieb-Liniger model. We have developed a variational approach based on a Lagrangian formalism to study the expansion for any regime of parameters. We have identified the possible physical situations at which self-similarity is violated. This should occur in a rather wide range of parameters $(0.01 \lesssim A \lesssim 100)$. The particular properties of the expansion of a gas in the strongly interacting regime could therefore be employed to discern between mean-field and strongly interacting regimes. In addition, the asymptotic behavior of the expanded cloud could be employed to discriminate between different initial interaction regimes of the system.

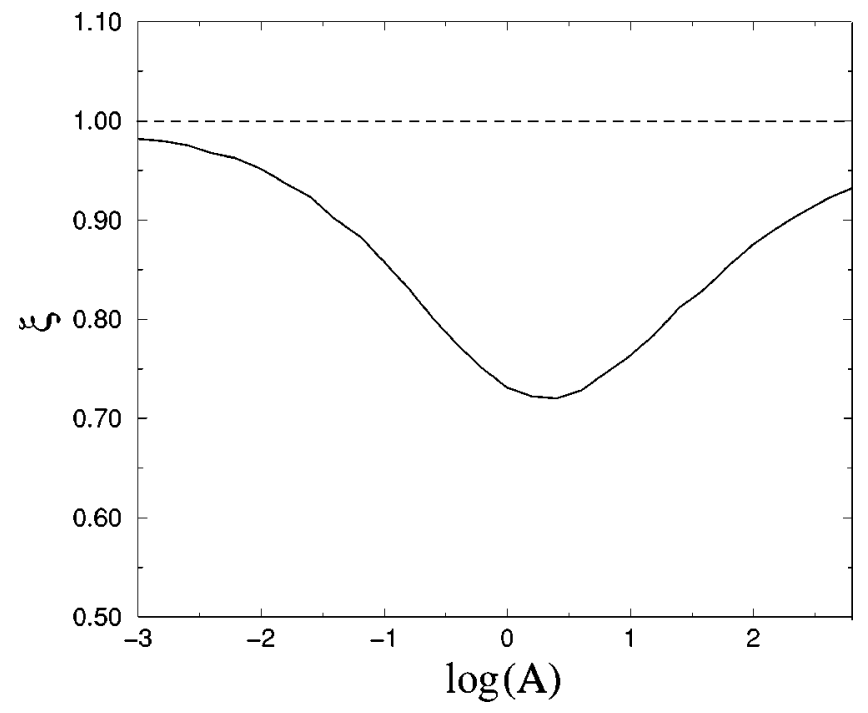

FIG. 5. Violation of the self-similarity. Value of $\xi=s_{\infty} / s_{0}$ as a function of the parameter $A$. The value $\xi=1$ denotes self-similarity.

Our discussion has been restricted to the analysis of the density properties. In fact the present formalism cannot describe the dynamics of the coherence in the system, i.e., we are limited to the diagonal terms of the corresponding singleparticle density matrix. The description of the nondiagonal terms lies beyond the scope of this paper, and requires other techniques of analysis $[19,32]$.

\section{ACKNOWLEDGMENTS}

We acknowledge support from Deutsche Forschungsgemeinschaft (SFB 407), the RTN Cold Quantum gases, ESF PESC BEC2000+, Royal Society of Edinburgh, and the Ministero dell'Istruzione, dell'Università e della Ricerca (MIUR). L.S. and P.P. acknowledge the Alexander von Humboldt Foundation, the Federal Ministry of Education and Research, and the ZIP Programme of the German Government. Discussions with M.D. Girardeau, M. Lewenstein, D.S. Petrov, and G.V. Shlyapnikov are acknowledged. We especially thank C. Menotti for providing us with the data of Ref. [18].
[1] M.H. Anderson et al., Science 269, 198 (1995); K.B. Davis et al., Phys. Rev. Lett. 75, 3969 (1995); C.C. Bradley et al., ibid. 78, 985 (1997).

[2] A. Görlitz et al., Phys. Rev. Lett. 87, 130402 (2001).

[3] A.I. Safonov et al., Phys. Rev. Lett. 81, 4545 (1998).

[4] W. Hänsel et al., Nature (London) 413, 498 (2001).

[5] S. Burger et al., Europhys. Lett. 57, 1 (2002).

[6] F. Schreck et al., Phys. Rev. Lett. 87, 080403 (2001).

[7] M. Greiner et al., Phys. Rev. Lett. 87, 160405 (2001).

[8] D.S. Petrov, G.V. Shlyapnikov, and J.T.M. Walraven, Phys. Rev. Lett. 85, 3745 (2000).

[9] Yu. Kagan et al., Phys. Rev. A 61, 043608 (2000).

[10] D.S. Petrov, M. Holzmann, and G.V. Shlyapnikov, Phys. Rev. Lett. 84, 2551 (2000).
[11] D.S. Petrov, G.V. Shlyapnikov, and J.T.M. Walraven, Phys. Rev. Lett. 87, 050404 (2001).

[12] S. Dettmer et al., Phys. Rev. Lett. 87, 160406 (2001); I. Shvarchuck et al., ibid. 89, 270404 (2002); F. Gerbier et al., Phys. Rev. A 67, 051602 (2003).

[13] M. Girardeau, J. Math. Phys. 1, 516 (1960).

[14] M. Olshanii, Phys. Rev. Lett. 81, 938 (1998).

[15] S. Cornish et al., Phys. Rev. Lett. 85, 1795 (2000); J. Stenger et al., ibid. 82, 2422 (1999).

[16] M. Greiner et al., Nature (London) 415, 39 (2002).

[17] V. Dunjko, V. Lorent, and M. Olshanii, Phys. Rev. Lett. 86, 5413 (2001).

[18] C. Menotti and S. Stringari, Phys. Rev. A 66, 043610 (2002).

[19] D.M. Gangardt and G.V. Shlyapnikov, Phys. Rev. Lett. 90, 010401 (2003). 
[20] P. Öhberg and L. Santos, Phys. Rev. Lett. 89, 240402 (2002).

[21] E.H. Lieb and W. Liniger, Phys. Rev. 130, 1605 (1963).

[22] C.N. Yang and C.P. Yang, J. Math. Phys. 10, 1115 (1969).

[23] M.D. Girardeau and E.M. Wright, Phys. Rev. Lett. 87, 210401 (2001); 84, 5691 (2000); 87, 050403 (2001); M.D. Girardeau, E.M. Wright, and J.M. Triscari, Phys. Rev. A 63, 033601 (2001).

[24] K.V. Kheruntsyan et al., Phys. Rev. Lett. 91, 040403 (2003).

[25] V.E. Korepin, N. M. Bogoliubov, and A.G. Izergin, Quantum Inverse Scattering Method and Correlation Functions (Cambridge University Press, Cambridge, 1993).

[26] E.P. Gross, Nuovo Cimento 20, 454 (1961); L.P. Pitaevskii, Sov. Phys. JETP 13, 451 (1961); E.P. Gross, J. Math. Phys. 4, 195 (1963).
[27] E.B. Kolomeisky et al., Phys. Rev. Lett. 85, 1146 (2000); E. Kolomiesky and J.P. Straley, Phys. Rev. B 46, 11749 (1992).

[28] Y. Castin and R. Dum, Phys. Rev. Lett. 77, 5315 (1996).

[29] Yu. Kagan, E.L. Surkov, and G.V. Shlyapnikov, Phys. Rev. A 54, R1753 (1996); 55, R18 (1997).

[30] F. Dalfovo et al., Phys. Lett. A 227, 259 (1997).

[31] Lagrangian (14) does not depend on $\dot{b}$ and $\dot{s}$, and consequently one obtains the dynamics only for $\alpha$ and $\beta$ imposing $\partial L / \partial b$ $=\partial L / \partial s=0$, and later on from these two conditions one can obtain the dynamics of $b$ and $s$. The gauge transformation (15) turns out to simplify significantly the calculations.

[32] G.E. Astrakharchik and S. Giorgini, Phys. Rev. Lett. (to be published), e-print cond-mat/0212512. 